\title{
Exact solutions for fractional partial differential equations by a new fractional sub-equation method
}

\author{
Bin Zheng ${ }^{*}$ and Chuanbao Wen
}

\section{"Correspondence:}

zhengbin2601@126.com

School of Science, Shandong

University of Technology, Zibo,

Shandong 255049, China

\begin{abstract}
In this paper, we propose a new fractional sub-equation method for finding exact solutions of fractional partial differential equations (FPDEs) in the sense of modified Riemann-Liouville derivative, which is the fractional version of the known $\left(\mathrm{G}^{\prime} / \mathrm{G}\right)$ method. To illustrate the validity of this method, we apply it to the space-time fractional Fokas equation, the space-time fractional $(2+1)$-dimensional dispersive long wave equations and the space-time fractional fifth-order Sawada-Kotera equation. As a result, some new exact solutions for them are successfully established.
\end{abstract} MSC: 35Q51; 35Q53

Keywords: fractional sub-equation method; fractional partial differential equations; exact solutions; fractional complex transformation

\section{Introduction}

Fractional differential equations are generalizations of classical differential equations of integer order. In recent decades, fractional differential equations have been the focus of many studies due to their frequent appearance in various applications in physics, biology, engineering, signal processing, systems identification, control theory, finance and fractional dynamics. Many articles have investigated some aspects of fractional differential equations such as the existence and uniqueness of solutions to Cauchy-type problems, the methods for explicit and numerical solutions, and the stability of solutions [1-8]. In [9], Jafari et al. applied the fractional sub-equation method to construct exact solutions of the fractional generalized reaction Duffing model and nonlinear fractional Sharma-TassoOlver equation. In [10], Baleanu et al. studied the existence and uniqueness of the solution for a nonlinear fractional differential equation boundary-value problem by using fixedpoint methods. In [11], Nyamoradi et al. investigated the existence of solutions for the multipoint boundary value problem of a fractional order differential inclusion.

Among the investigations for fractional differential equations, research into seeking exact solutions and numerical solutions of fractional differential equations is an important topic. Many powerful and efficient methods have been proposed to obtain numerical solutions and exact solutions of fractional differential equations so far. For example, these methods include the Adomian decomposition method [12-14], the variational iterative method [15-22], the homotopy perturbation method [23-26], the differential transformation method [27], the finite difference method [28], the finite element method [29], 
the fractional Riccati sub-equation method [30-32] and so on. In these investigations, we note that many authors have sought exact and numerical solutions for fractional partial differential equations (FPDEs) in the sense of modified Riemann-Liouville derivative (for example, see $[16,17,30-34])$. Based on these methods, a variety of fractional differential equations have been investigated.

In this paper, we propose a new fractional sub-equation method to establish exact solutions for fractional partial differential equations (FPDEs) in the sense of modified Riemann-Liouville derivative defined by Jumarie [35], which is a fractional version of the known $\left(G^{\prime} / G\right)$ method [36-39]. This method is based on the following fractional ODE:

$$
D_{\xi}^{2 \alpha} G(\xi)+\lambda D_{\xi}^{\alpha} G(\xi)+\mu G(\xi)=0
$$

where $D_{\xi}^{\alpha} G(\xi)$ denotes the modified Riemann-Liouville derivative of order $\alpha$ for $G(\xi)$ with respect to $\xi$.

The rest of this paper is organized as follows. In Section 2, we present some definitions and properties of Jumarie's modified Riemann-Liouville derivative and the expression for $\frac{D_{\xi}^{\alpha} G(\xi)}{G(\xi)}$ related to Eq. (1). In Section 3, we give the description of the fractional sub-equation method for solving FPDEs. Then in Section 4 we apply this method to establish exact solutions for the space-time fractional Fokas equation, the space-time fractional $(2+1)$-dimensional dispersive long wave equations and the space-time fractional fifth-order Sawada-Kotera equation. Some conclusions are presented at the end of the paper.

2 Jumarie's modified Riemann-Liouville derivative and general expression for $\frac{D_{\xi}^{\alpha} G(\xi)}{G(\xi)}$

Jumarie's modified Riemann-Liouville derivative of order $\alpha$ is defined by the following expression [35]:

$$
D_{t}^{\alpha} f(t)= \begin{cases}\frac{1}{\Gamma(1-\alpha)} \frac{d}{d t} \int_{0}^{t}(t-\xi)^{-\alpha}(f(\xi)-f(0)) d \xi, & 0<\alpha<1, \\ \left(f^{(n)}(t)\right)^{(\alpha-n)}, & n \leq \alpha<n+1, n \geq 1 .\end{cases}
$$

We list some important properties for the modified Riemann-Liouville derivative as follows (see [35, Eqs. (3.10)-(3.13)]):

$$
\begin{aligned}
& D_{t}^{\alpha} t^{r}=\frac{\Gamma(1+r)}{\Gamma(1+r-\alpha)} t^{r-\alpha}, \\
& D_{t}^{\alpha}(f(t) g(t))=g(t) D_{t}^{\alpha} f(t)+f(t) D_{t}^{\alpha} g(t), \\
& D_{t}^{\alpha} f[g(t)]=f_{g}^{\prime}[g(t)] D_{t}^{\alpha} g(t)=D_{g}^{\alpha} f[g(t)]\left(g^{\prime}(t)\right)^{\alpha} .
\end{aligned}
$$

In order to obtain the general solutions for Eq. (1), we suppose $G(\xi)=H(\eta)$ and a nonlinear fractional complex transformation $\eta=\frac{\xi^{\alpha}}{\Gamma(1+\alpha)}$. Then by Eq. (2) and the first equality in Eq. (4), Eq. (1) can be turned into the following second ordinary differential equation

$$
H^{\prime \prime}(\eta)+\lambda H^{\prime}(\eta)+\mu H(\eta)=0
$$


By the general solutions of Eq. (5), we have

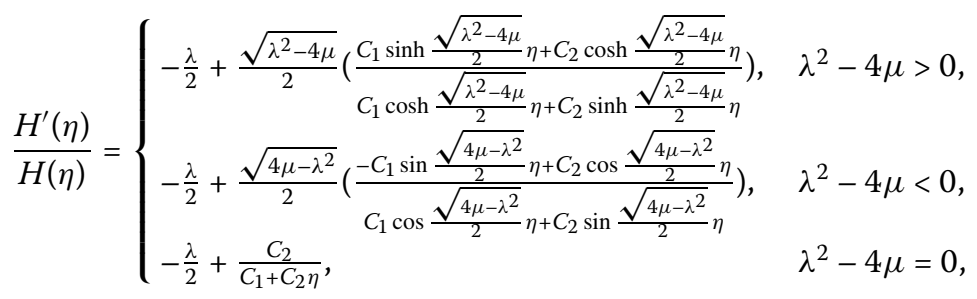

where $C_{1}, C_{2}$ are arbitrary constants.

Since $D_{\xi}^{\alpha} G(\xi)=D_{\xi}^{\alpha} H(\eta)=H^{\prime}(\eta) D_{\xi}^{\alpha} \eta=H^{\prime}(\eta)$, we obtain

$$
\frac{D_{\xi}^{\alpha} G(\xi)}{G(\xi)}=\left\{\begin{array}{cl}
-\frac{\lambda}{2}+\frac{\sqrt{\lambda^{2}-4 \mu}}{2}\left(\frac{C_{1} \sinh \frac{\sqrt{\lambda^{2}-4 \mu}}{2 \Gamma(1+\alpha)} \xi^{\alpha}+C_{2} \cosh \frac{\sqrt{\lambda^{2}-4 \mu}}{2 \Gamma(1+\alpha)} \xi^{\alpha}}{C_{1} \cosh \frac{\sqrt{\lambda^{2}-4 \mu}}{2 \Gamma(1+\alpha)} \xi^{\alpha}+C_{2} \sinh \frac{\sqrt{\lambda^{2}-4 \mu}}{2 \Gamma(1+\alpha)} \xi^{\alpha}}\right), & \lambda^{2}-4 \mu>0, \\
-\frac{\lambda}{2}+\frac{\sqrt{4 \mu-\lambda^{2}}}{2}\left(\frac{-C_{1} \sin \frac{\sqrt{4 \mu-\lambda^{2}}}{2 \Gamma(1+\alpha)} \xi^{\alpha}+C_{2} \cos \frac{\sqrt{4 \mu-\lambda^{2}}}{2 \Gamma(1+\alpha)} \xi^{\alpha}}{C_{1} \cos \frac{\sqrt{4 \mu-\lambda^{2}}}{2 \Gamma(1+\alpha)} \xi^{\alpha}+C_{2} \sin \frac{\sqrt{4 \mu-\lambda^{2}}}{2 \Gamma(1+\alpha)} \xi^{\alpha}}\right), & \lambda^{2}-4 \mu<0, \\
-\frac{\lambda}{2}+\frac{C_{2} \Gamma(1+\alpha)}{C_{1} \Gamma(1+\alpha)+C_{2} \xi^{\alpha}}, & \lambda^{2}-4 \mu=0 .
\end{array}\right.
$$

\section{Description of the fractional sub-equation method}

In this section we describe the main steps of the fractional sub-equation method for finding exact solutions of FPDEs.

Suppose that a fractional partial differential equation, say in the independent variables $t, x_{1}, x_{2}, \ldots, x_{n}$, is given by

$$
\begin{aligned}
& P\left(u_{1}, \ldots u_{k}, D_{t}^{\alpha} u_{1}, \ldots, D_{t}^{\alpha} u_{k}, D_{x_{1}}^{\alpha} u_{1}, \ldots, D_{x_{1}}^{\alpha} u_{k}, \ldots,\right. \\
& \left.D_{x_{n}}^{\alpha} u_{1}, \ldots, D_{x_{n}}^{\alpha} u_{k}, D_{t}^{2 \alpha} u_{1}, \ldots, D_{t}^{2 \alpha} u_{k}, D_{x_{1}}^{2 \alpha} u_{1}, \ldots\right)=0,
\end{aligned}
$$

where $u_{i}=u_{i}\left(t, x_{1}, x_{2}, \ldots, x_{n}\right), i=1, \ldots, k$, are unknown functions, $P$ is a polynomial in $u_{i}$ and their various partial derivatives include fractional derivatives.

Step 1. Suppose that

$$
u_{i}\left(t, x_{1}, x_{2}, \ldots, x_{n}\right)=U_{i}(\xi), \quad \xi=c t+k_{1} x_{1}+k_{2} x_{2}+\cdots+k_{n} x_{n}+\xi_{0} .
$$

Then by the second equality in Eq. (4), Eq. (8) can be turned into the following fractional ordinary differential equation with respect to the variable $\xi$ :

$$
\begin{gathered}
\widetilde{P}\left(U_{1}, \ldots, U_{k}, c^{\alpha} D_{\xi}^{\alpha} U_{1}, \ldots, c^{\alpha} D_{\xi}^{\alpha} U_{k}, k_{1}^{\alpha} D_{\xi}^{\alpha} U_{1}, \ldots, k_{1}^{\alpha} D_{\xi}^{\alpha} U_{k}, \ldots, k_{n}^{\alpha} D_{\xi}^{\alpha} U_{1}, \ldots,\right. \\
\left.k_{n}^{\alpha} D_{\xi}^{\alpha} U_{k}, c^{2 \alpha} D_{\xi}^{2 \alpha} U_{1}, \ldots, c^{2 \alpha} D_{\xi}^{2 \alpha} U_{k}, k_{1}^{2 \alpha} D_{\xi}^{2 \alpha} U_{1}, \ldots\right)=0 .
\end{gathered}
$$

Step 2. Suppose that the solution of (10) can be expressed by a polynomial in $\left(\frac{D_{\xi}^{\alpha} G}{G}\right)$ as follows:

$$
U_{j}(\xi)=\sum_{i=0}^{m_{j}} a_{j, i}\left(\frac{D_{\xi}^{\alpha} G}{G}\right)^{i}, \quad j=1,2, \ldots, k
$$


where $G=G(\xi)$ satisfies Eq. (1), and $a_{j, i}, i=0,1, \ldots, m, j=1,2, \ldots, k$, are constants to be determined later with $a_{j, m} \neq 0$. The positive integer $m$ can be determined by considering the homogeneous balance between the highest order derivatives and nonlinear terms appearing in (10).

Step 3. Substituting (11) into (10) and using (1), collecting all terms with the same order of $\left(\frac{D_{\xi}^{\alpha} G}{G}\right)$ together, the left-hand side of $(10)$ is converted into another polynomial in $\left(\frac{D_{\xi}^{\alpha} G}{G}\right)$. Equating each coefficient of this polynomial to zero yields a set of algebraic equations for $a_{j, i}, i=0,1, \ldots, m, j=1,2, \ldots, k$.

Step 4. Solving the equation system in Step 3 and using (7), we can construct a variety of exact solutions for Eq. (8).

Remark 1 If we set $\alpha=1$ in Eq. (1), then it becomes $G^{\prime \prime}(\xi)+\lambda G^{\prime}(\xi)+\mu G(\xi)=0$, which is the foundation of the known $\left(G^{\prime} / G\right)$ method for solving partial differential equations (PDEs). So, in this way, the described fractional sub-equation method above is the extension of the $\left(G^{\prime} / G\right)$ method to fractional case.

Remark 2 The idea of the transformation from $n$ independent variables to one independent variable denoted in Eq. (9) is similar to that in [30, Eq. (12)], [31, Eq. (8)], and [33, Eq. (6)]. After applying this transformation to Eq. (8), by use of the second equality of Eq. (4), the original fractional partial differential equation can be transformed into another fractional ordinary differential equation in one independent variable.

\section{Applications of the method}

\subsection{Space-time fractional Fokas equation}

We consider the space-time fractional Fokas equation

$$
\begin{aligned}
& 4 \frac{\partial^{2 \alpha} q}{\partial t^{\alpha} \partial x_{1}^{\alpha}}-\frac{\partial^{4 \alpha} q}{\partial x_{1}^{3 \alpha} \partial x_{2}^{\alpha}}+\frac{\partial^{4 \alpha} q}{\partial x_{2}^{3 \alpha} \partial x_{1}^{\alpha}}+12 \frac{\partial^{\alpha} q}{\partial x_{1}^{\alpha}} \frac{\partial^{\alpha} q}{\partial x_{2}^{\alpha}}+12 q \frac{\partial^{2 \alpha} q}{\partial x_{1}^{\alpha} \partial x_{2}^{\alpha}}-6 \frac{\partial^{2 \alpha} q}{\partial y_{1}^{\alpha} \partial y_{2}^{\alpha}} \\
& \quad=0, \quad 0<\alpha \leq 1 .
\end{aligned}
$$

In [30], the authors solved Eq. (12) by a fractional Riccati sub-equation method and obtained some exact solutions for it. Now we will apply the method described in Section 3 to Eq. (12).

Suppose that $q(x, y, t)=U(\xi)$, where $\xi=c t+k_{1} x_{1}+k_{2} x_{2}+l_{1} y_{1}+l_{2} y_{2}+\xi_{0}, k_{1}, k_{2}, l_{1}, l_{2}, c$, $\xi_{0}$ are all constants with $k_{1}, k_{2}, l_{1}, l_{2}, c \neq 0$. Then by use of the second equality in Eq. (4), Eq. (12) can be turned into

$$
\begin{aligned}
& 4 c^{\alpha} k_{1}^{\alpha} D_{\xi}^{2 \alpha} U-k_{1}^{3 \alpha} k_{2}^{\alpha} D_{\xi}^{4 \alpha} U+k_{2}^{3 \alpha} k_{1}^{\alpha} D_{\xi}^{4 \alpha} U \\
& \quad+12 k_{1}^{\alpha} k_{2}^{\alpha}\left(D_{\xi}^{\alpha} U\right)^{2}+12 k_{1}^{\alpha} k_{2}^{\alpha} U D_{\xi}^{2 \alpha} U-6 l_{1}^{\alpha} l_{2}^{\alpha} D_{\xi}^{2 \alpha} U=0 .
\end{aligned}
$$

Suppose that the solution of Eq. (13) can be expressed by

$$
U(\xi)=\sum_{i=0}^{m} a_{i}\left(\frac{D_{\xi}^{\alpha} G}{G}\right)^{i}
$$


where $G=G(\xi)$ satisfies Eq. (1). By balancing the order between the highest order derivative term and nonlinear term in Eq. (13), we can obtain $m=2$. So, we have

$$
U(\xi)=a_{0}+a_{1}\left(\frac{D_{\xi}^{\alpha} G}{G}\right)+a_{2}\left(\frac{D_{\xi}^{\alpha} G}{G}\right)^{2}
$$

Substituting (15) into (13) and collecting all the terms with the same power of $\left(\frac{D_{\xi}^{\alpha} G}{G}\right)$ together, equating each coefficient to zero, yield a set of algebraic equations. Solving these equations yields

$$
\begin{aligned}
& a_{0}=\frac{k_{1}^{3 \alpha} k_{2}^{\alpha} \lambda^{2}-k_{1}^{\alpha} k_{2}^{3 \alpha} \lambda^{2}+8 k_{1}^{3 \alpha} k_{2}^{\alpha} \mu-8 k_{1}^{\alpha} k_{2}^{3 \alpha} \mu-4 c^{\alpha} k_{1}^{\alpha}+6 l_{1}^{\alpha} l_{2}^{\alpha}}{12 k_{1}^{\alpha} k_{2}^{\alpha}} \\
& a_{1}=\lambda\left(k_{1}^{2 \alpha}-k_{2}^{2 \alpha}\right) \\
& a_{2}=k_{1}^{2 \alpha}-k_{2}^{2 \alpha} .
\end{aligned}
$$

Substituting the result above into Eq. (15) and combining with (7), we can obtain the following exact solutions to Eq. (12).

When $\lambda^{2}-4 \mu>0$,

$$
\begin{aligned}
q_{1}\left(t, x_{1}, x_{2}, y_{1}, y_{2}\right) \\
=\frac{k_{1}^{3 \alpha} k_{2}^{\alpha} \lambda^{2}-k_{1}^{\alpha} k_{2}^{3 \alpha} \lambda^{2}+8 k_{1}^{3 \alpha} k_{2}^{\alpha} \mu-8 k_{1}^{\alpha} k_{2}^{3 \alpha} \mu-4 c^{\alpha} k_{1}^{\alpha}+6 l_{1}^{\alpha} l_{2}^{\alpha}}{12 k_{1}^{\alpha} k_{2}^{\alpha}} \\
\quad+\lambda\left(k_{1}^{2 \alpha}-k_{2}^{2 \alpha}\right)\left[-\frac{\lambda}{2}+\frac{\sqrt{\lambda^{2}-4 \mu}}{2}\left(\frac{C_{1} \sinh \frac{\sqrt{\lambda^{2}-4 \mu}}{2 \Gamma(1+\alpha)} \xi^{\alpha}+C_{2} \cosh \frac{\sqrt{\lambda^{2}-4 \mu}}{2 \Gamma(1+\alpha)} \xi^{\alpha}}{C_{1} \cosh \frac{\sqrt{\lambda^{2}-4 \mu}}{2 \Gamma(1+\alpha)} \xi^{\alpha}+C_{2} \sinh \frac{\sqrt{\lambda^{2}-4 \mu}}{2 \Gamma(1+\alpha)} \xi^{\alpha}}\right)\right] \\
\quad+\left(k_{1}^{2 \alpha}-k_{2}^{2 \alpha}\right)\left[-\frac{\lambda}{2}+\frac{\sqrt{\lambda^{2}-4 \mu}}{2}\left(\frac{C_{1} \sinh \frac{\sqrt{\lambda^{2}-4 \mu}}{2 \Gamma(1+\alpha)} \xi^{\alpha}+C_{2} \cosh \frac{\sqrt{\lambda^{2}-4 \mu}}{2 \Gamma(1+\alpha)} \xi^{\alpha}}{C_{1} \cosh \frac{\sqrt{\lambda^{2}-4 \mu}}{2 \Gamma(1+\alpha)} \xi^{\alpha}+C_{2} \sinh \frac{\sqrt{\lambda^{2}-4 \mu}}{2 \Gamma(1+\alpha)} \xi^{\alpha}}\right)\right]^{2},
\end{aligned}
$$

where $\xi=c t+k_{1} x_{1}+k_{2} x_{2}+l_{1} y_{1}+l_{2} y_{2}+\xi_{0}$.

When $\lambda^{2}-4 \mu<0$,

$$
\begin{aligned}
q_{2}( & \left.t, x_{1}, x_{2}, y_{1}, y_{2}\right) \\
= & \frac{k_{1}^{3 \alpha} k_{2}^{\alpha} \lambda^{2}-k_{1}^{\alpha} k_{2}^{3 \alpha} \lambda^{2}+8 k_{1}^{3 \alpha} k_{2}^{\alpha} \mu-8 k_{1}^{\alpha} k_{2}^{3 \alpha} \mu-4 c^{\alpha} k_{1}^{\alpha}+6 l_{1}^{\alpha} l_{2}^{\alpha}}{12 k_{1}^{\alpha} k_{2}^{\alpha}} \\
& +\lambda\left(k_{1}^{2 \alpha}-k_{2}^{2 \alpha}\right)\left[-\frac{\lambda}{2}+\frac{\sqrt{4 \mu-\lambda^{2}}}{2}\left(\frac{-C_{1} \sin \frac{\sqrt{4 \mu-\lambda^{2}}}{2 \Gamma(1+\alpha)} \xi^{\alpha}+C_{2} \cos \frac{\sqrt{4 \mu-\lambda^{2}}}{2 \Gamma(1+\alpha)} \xi^{\alpha}}{C_{1} \cos \frac{\sqrt{4 \mu-\lambda^{2}}}{2 \Gamma(1+\alpha)} \xi^{\alpha}+C_{2} \sin \frac{\sqrt{4 \mu-\lambda^{2}}}{2 \Gamma(1+\alpha)} \xi^{\alpha}}\right)\right] \\
& +\left(k_{1}^{2 \alpha}-k_{2}^{2 \alpha}\right)\left[-\frac{\lambda}{2}+\frac{\sqrt{4 \mu-\lambda^{2}}}{2}\left(\frac{-C_{1} \sin \frac{\sqrt{4 \mu-\lambda^{2}}}{2 \Gamma(1+\alpha)} \xi^{\alpha}+C_{2} \cos \frac{\sqrt{4 \mu-\lambda^{2}}}{2 \Gamma(1+\alpha)} \xi^{\alpha}}{C_{1} \cos \frac{\sqrt{4 \mu-\lambda^{2}}}{2 \Gamma(1+\alpha)} \xi^{\alpha}+C_{2} \sin \frac{\sqrt{4 \mu-\lambda^{2}}}{2 \Gamma(1+\alpha)} \xi^{\alpha}}\right)\right]^{2},
\end{aligned}
$$

where $\xi=c t+k_{1} x_{1}+k_{2} x_{2}+l_{1} y_{1}+l_{2} y_{2}+\xi_{0}$. 
When $\lambda^{2}-4 \mu=0$,

$$
\begin{aligned}
q_{3}( & \left.t, x_{1}, x_{2}, y_{1}, y_{2}\right) \\
= & \frac{k_{1}^{3 \alpha} k_{2}^{\alpha} \lambda^{2}-k_{1}^{\alpha} k_{2}^{3 \alpha} \lambda^{2}+8 k_{1}^{3 \alpha} k_{2}^{\alpha} \mu-8 k_{1}^{\alpha} k_{2}^{3 \alpha} \mu-4 c^{\alpha} k_{1}^{\alpha}+6 l_{1}^{\alpha} l_{2}^{\alpha}}{12 k_{1}^{\alpha} k_{2}^{\alpha}} \\
& +\lambda\left(k_{1}^{2 \alpha}-k_{2}^{2 \alpha}\right)\left[-\frac{\lambda}{2}+\frac{C_{2} \Gamma(1+\alpha)}{C_{1} \Gamma(1+\alpha)+C_{2} \xi^{\alpha}}\right] \\
& +\left(k_{1}^{2 \alpha}-k_{2}^{2 \alpha}\right)\left[-\frac{\lambda}{2}+\frac{C_{2} \Gamma(1+\alpha)}{C_{1} \Gamma(1+\alpha)+C_{2} \xi^{\alpha}}\right]^{2},
\end{aligned}
$$

where $\xi=c t+k_{1} x_{1}+k_{2} x_{2}+l_{1} y_{1}+l_{2} y_{2}+\xi_{0}$.

Remark 3 As one can see, the established solutions for the space-time fractional Fokas equation above are different from the results in [30] and are new exact solutions so far to our best knowledge.

Remark 4 The method used above in solving Eq. (12) can also be used to obtain solutions to initial or boundary value problems. For example, if we add the initial value condition

$$
q\left(0, x_{1}, x_{2}, y_{1}, y_{2}\right)=\tan ^{2}\left(\frac{\sqrt{2 x_{1}+x_{2}+y_{1}+y_{2}}}{\Gamma(1.5)}\right)
$$

then, after substituting this initial value condition to the trigonometric solutions obtained in Eq. (17) and fulfilling some basic comparison and computation, one can see that $\alpha=0.5$, $k_{1}=2, k_{2}=1, l_{1}=l_{2}=1, \xi_{0}=0, C_{2}=0, \lambda=0, \mu=1, c=\frac{41}{8}+3 \sqrt{2}$. So, we obtain the solution to the initial value problem as

$$
q\left(t, x_{1}, x_{2}, y_{1}, y_{2}\right)=\tan ^{2}\left(\frac{\sqrt{2 x_{1}+x_{2}+y_{1}+y_{2}+\left(\frac{41}{8}+3 \sqrt{2}\right) t}}{\Gamma(1.5)}\right) .
$$

\subsection{Space-time fractional $(2+1)$-dimensional dispersive long wave equations}

We consider the following space-time fractional $(2+1)$-dimensional dispersive long wave equations

$$
\left\{\begin{array}{l}
\frac{\partial^{2 \alpha} u}{\partial y^{\alpha} \partial t^{\alpha}}+\frac{\partial^{2 \alpha} v}{\partial x^{2 \alpha}}+\frac{\partial^{\alpha}\left(u \frac{\partial^{\alpha} u}{\partial x^{\alpha}}\right)}{\partial y^{\alpha}}=0, \\
\frac{\partial^{\alpha} v}{\partial t^{\alpha}}+\frac{\partial^{\alpha} u}{\partial x^{\alpha}}+\frac{\partial^{\alpha}(u v)}{\partial x^{\alpha}}+\frac{\partial^{3 \alpha} u}{\partial x^{2 \alpha} \partial y^{\alpha}}=0,
\end{array}\right.
$$

which are the known $(2+1)$-dimensional dispersive long wave equations [40-53]:

$$
\left\{\begin{array}{l}
u_{y t}+v_{x x}+\left(u u_{x}\right)_{y}=0 \\
v_{t}+u_{x}+(u v)_{x}+u_{x x y}=0
\end{array}\right.
$$

Some types of exact solutions for Eqs. (20) have been obtained in [40-53] by use of various methods including the Riccati sub-equation method $[40,41,46]$, the nonlinear transformation method [42], the Jacobi function method [44, 45, 53], the ( $\left.G^{\prime} / G\right)$-expansion method [43], the modified CK's direct method [47], the EXP-function method [48], 
the Hopf-Cole transformation method [49], the modified extended Fan's sub-equation method [50, 51], the generalized algebraic method [52]. But we notice that so far no research has been pursued for Eqs. (19). In the following, we will apply the proposed fractional sub-equation method to Eqs. (19).

To begin with, we suppose $u(x, y, t)=U(\xi), v(x, y, t)=V(\xi)$, where $\xi=k_{1} x+k_{2} y+c t+\xi_{0}$, $k_{1}, k_{2}, c, \xi_{0}$ are all constants with $k_{1}, k_{2}, c \neq 0$. Then by use of the second equality in Eq. (4), Eqs. (19) can be turned into

$$
\left\{\begin{array}{l}
c^{\alpha} k_{2}^{\alpha} D_{\xi}^{2 \alpha} U+k_{1}^{2 \alpha} D_{\xi}^{2 \alpha} V+k_{1}^{\alpha} k_{2}^{\alpha}\left(U D_{\xi}^{2 \alpha} U+\left(D_{\xi}^{\alpha} U\right)^{2}\right)=0 \\
c^{\alpha} D_{\xi}^{\alpha} V+k_{1}^{\alpha} D_{\xi}^{\alpha} U+k_{1}^{\alpha} D_{\xi}^{\alpha}(U V)+k_{1}^{2 \alpha} k_{2}^{\alpha} D_{\xi}^{3 \alpha} U=0
\end{array}\right.
$$

Suppose that the solution of Eqs. (21) can be expressed by

$$
\left\{\begin{array}{l}
U(\xi)=\sum_{i=0}^{m_{1}} a_{i}\left(\frac{D_{\xi}^{\alpha} G}{G}\right)^{i} \\
V(\xi)=\sum_{i=0}^{m_{2}} b_{i}\left(\frac{D_{\xi}^{\alpha} G}{G}\right)^{i}
\end{array}\right.
$$

Balancing the order of $D_{\xi}^{2 \alpha} V$ and $U D_{\xi}^{2 \alpha} U, D_{\xi}^{\alpha}(U V)$ and $D_{\xi}^{3 \alpha} U$ in (21), we can obtain $m_{1}=1$, $m_{2}=2$. So, we have

$$
\left\{\begin{array}{l}
U(\xi)=a_{0}+a_{1}\left(\frac{D_{\xi}^{\alpha} G}{G}\right), \\
V(\xi)=b_{0}+b_{1}\left(\frac{D_{\xi}^{\alpha} G}{G}\right)+b_{2}\left(\frac{D_{\xi}^{\alpha} G}{G}\right)^{2} .
\end{array}\right.
$$

Substituting (23) into (21), using Eq. (1) and collecting all the terms with the same power of $\left(\frac{D_{\xi}^{\alpha} G}{G}\right)$ together, equating each coefficient to zero, yield a set of algebraic equations. Solving these equations yields

$$
\begin{aligned}
& a_{0}=\frac{ \pm k_{1}^{2 \alpha} \lambda-c^{\alpha}}{k_{1}^{\alpha}}, \quad a_{1}= \pm 2 k_{1}^{\alpha}, \quad b_{0}=-2 k_{1}^{\alpha} k_{2}^{\alpha} \mu-1, \\
& b_{1}=-2 k_{1}^{\alpha} k_{2}^{\alpha} \lambda, \quad b_{2}=-2 k_{1}^{\alpha} k_{2}^{\alpha} .
\end{aligned}
$$

Substituting the result above into Eq. (23) and combining with (7), we can obtain the following exact solutions to Eqs. (19).

When $\lambda^{2}-4 \mu>0$,

$$
\left\{\begin{aligned}
& u_{1}(x, y, t)= \frac{ \pm k_{1}^{2 \alpha} \lambda-c^{\alpha}}{k_{1}^{\alpha}} \pm 2 k_{1}^{\alpha}\left[-\frac{\lambda}{2}+\frac{\sqrt{\lambda^{2}-4 \mu}}{2}\left(\frac{C_{1} \sinh \frac{\sqrt{\lambda^{2}-4 \mu}}{2 \Gamma(1+\alpha)} \xi^{\alpha}+C_{2} \cosh \frac{\sqrt{\lambda^{2}-4 \mu}}{2 \Gamma(1+\alpha)} \xi^{\alpha}}{C_{1} \cosh \frac{\sqrt{\lambda^{2}-4 \mu}}{2 \Gamma(1+\alpha)} \xi^{\alpha}+C_{2} \sinh \frac{\sqrt{\lambda^{2}-4 \mu}}{2 \Gamma(1+\alpha)} \xi^{\alpha}}\right)\right] \\
&-\frac{3}{2} k_{1}^{2 \alpha}\left[-\frac{\lambda}{2}+\frac{\sqrt{\lambda^{2}-4 \mu}}{2}\left(\frac{C_{1} \sinh \frac{\sqrt{\lambda^{2}-4 \mu}}{2 \Gamma(1+\alpha)} \xi^{\alpha}+C_{2} \cosh \frac{\sqrt{\lambda^{2}-4 \mu}}{2 \Gamma(1+\alpha)} \xi^{\alpha}}{C_{1} \cosh \frac{\sqrt{\lambda^{2}-4 \mu}}{2 \Gamma(1+\alpha)} \xi^{\alpha}+C_{2} \sinh \frac{\sqrt{\lambda^{2}-4 \mu}}{2 \Gamma(1+\alpha)} \xi^{\alpha}}\right)\right. \\
& v_{1}(x, y, t)=-2 k_{1}^{\alpha} k_{2}^{\alpha} \mu-1 \\
&-2 k_{1}^{\alpha} k_{2}^{\alpha} \lambda\left[-\frac{\lambda}{2}+\frac{\sqrt{\lambda^{2}-4 \mu}}{2}\left(\frac{C_{1} \sinh \frac{\sqrt{\lambda^{2}-4 \mu}}{2 \Gamma(1+\alpha)} \xi^{\alpha}+C_{2} \cosh \frac{\sqrt{\lambda^{2}-4 \mu}}{2 \Gamma(1+\alpha)} \xi^{\alpha}}{C_{1} \cosh \frac{\sqrt{\lambda^{2}-4 \mu}}{2 \Gamma(1+\alpha)} \xi^{\alpha}+C_{2} \sinh \frac{\sqrt{\lambda^{2}-4 \mu}}{2 \Gamma(1+\alpha)} \xi^{\alpha}}\right)\right] \\
&-2 k_{1}^{\alpha} k_{2}^{\alpha}\left[-\frac{\lambda}{2}+\frac{\sqrt{\lambda^{2}-4 \mu}}{2}\left(\frac{C_{1} \sinh \frac{\sqrt{\lambda^{2}-4 \mu}}{2 \Gamma(1+\alpha)} \xi^{\alpha}+C_{2} \cosh \frac{\sqrt{\lambda^{2}-4 \mu}}{2 \Gamma(1+\alpha)} \xi^{\alpha}}{2 \sqrt{\lambda^{2}-4 \mu}}\right)\right]^{2}, \\
& C_{1} \cosh \frac{\sqrt{2} \frac{1(1+\alpha)}{2 \Gamma}+C_{2} \sinh \frac{\sqrt{\lambda^{2}-4 \mu}}{2 \Gamma(1+\alpha)} \xi^{\alpha}}{2 \Gamma(1+\alpha)}
\end{aligned}\right.
$$

where $\xi=k_{1} x+k_{2} y+c t+\xi_{0}$. 
When $\lambda^{2}-4 \mu<0$,

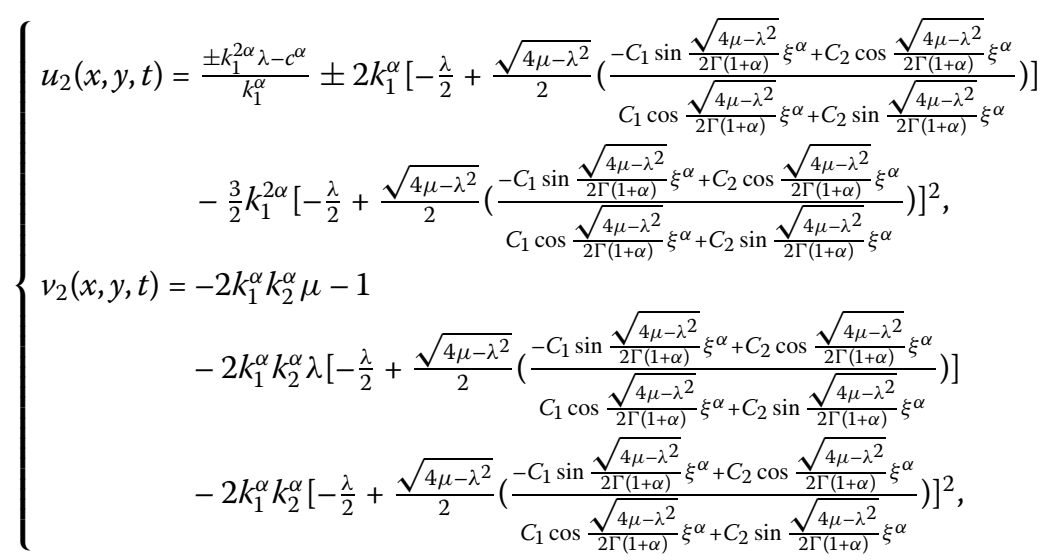

where $\xi=k_{1} x+k_{2} y+c t+\xi_{0}$.

When $\lambda^{2}-4 \mu=0$,

$$
\left\{\begin{aligned}
u_{3}(x, y, t)= & \frac{ \pm k_{1}^{2 \alpha} \lambda-c^{\alpha}}{k_{1}^{\alpha}} \pm 2 k_{1}^{\alpha}\left[-\frac{\lambda}{2}+\frac{C_{2} \Gamma(1+\alpha)}{C_{1} \Gamma(1+\alpha)+C_{2} \xi^{\alpha}}\right]-\frac{3}{2} k_{1}^{2 \alpha}\left[-\frac{\lambda}{2}+\frac{C_{2} \Gamma(1+\alpha)}{C_{1} \Gamma(1+\alpha)+C_{2} \xi^{\alpha}}\right]^{2} \\
v_{3}(x, y, t)= & -2 k_{1}^{\alpha} k_{2}^{\alpha} \mu-1-2 k_{1}^{\alpha} k_{2}^{\alpha} \lambda\left[-\frac{\lambda}{2}+\frac{C_{2} \Gamma(1+\alpha)}{C_{1} \Gamma(1+\alpha)+C_{2} \xi^{\alpha}}\right] \\
& -2 k_{1}^{\alpha} k_{2}^{\alpha}\left[-\frac{\lambda}{2}+\frac{C_{2} \Gamma(1+\alpha)}{C_{1} \Gamma(1+\alpha)+C_{2} \xi^{\alpha}}\right]^{2}
\end{aligned}\right.
$$

where $\xi=k_{1} x+k_{2} y+c t+\xi_{0}$.

Remark 5 The established solutions in Eqs. (24)-(26) are new exact solutions for the space-time fractional $(2+1)$-dimensional dispersive long wave equations.

\subsection{Space-time fractional fifth-order Sawada-Kotera equation}

We consider the space-time fractional fifth-order Sawada-Kotera equation

$$
D_{t}^{\alpha} u+D_{x}^{5 \alpha} u+45 u^{2} D_{x}^{\alpha} u+15\left(D_{x}^{\alpha} u D_{x}^{2 \alpha} u+u D_{x}^{3 \alpha} u\right)=0, \quad 0<\alpha \leq 1,
$$

which is the variation of the fifth-order Sawada-Kotera equation [54]

$$
u_{t}+u_{x x x x x}+45 u_{x} u^{2}+15\left(u_{x} u_{x x}+u u_{x x x}\right)=0
$$

Now we apply the proposed method to Eq. (27). To begin with, we suppose $u(x, t)=U(\xi)$, where $\xi=k x+c t+\xi_{0}, k, c, \xi_{0}$ are all constants with $k, c \neq 0$. Then, by use of the second equality in Eq. (4), Eq. (27) can be turned into

$$
c^{\alpha} D_{\xi}^{\alpha} U+k^{5 \alpha} D_{\xi}^{5 \alpha} U+45 k^{\alpha} U D_{\xi}^{\alpha} U+15 k^{3 \alpha}\left(D_{\xi}^{\alpha} U D_{\xi}^{2 \alpha} U+U D_{\xi}^{3 \alpha} U\right)=0 .
$$

Suppose that the solution of Eq. (29) can be expressed by

$$
U(\xi)=\sum_{i=0}^{m} a_{i}\left(\frac{D_{\xi}^{\alpha} G}{G}\right)^{i}
$$


Balancing the order of $U^{(5)}$ and $U D_{\xi}^{3 \alpha} U$ in Eq. (30), we have $m=2$. So,

$$
U(\xi)=a_{0}+a_{1}\left(\frac{D_{\xi}^{\alpha} G}{G}\right)+a_{2}\left(\frac{D_{\xi}^{\alpha} G}{G}\right)^{2}
$$

Substituting (31) into (29), using Eq. (1) and collecting all the terms with the same power of $\left(\frac{D_{\xi}^{\alpha} G}{G}\right)$ together, equating each coefficient to zero, yield a set of algebraic equations. Solving these equations yields

$$
\begin{aligned}
& a_{0}=-\frac{40 k^{3 \alpha} \mu+5 k^{3 \alpha} \lambda^{2} \pm \sqrt{80 k^{6 \alpha} \mu^{2}-40 k^{6 \alpha} \mu \lambda^{2}+5 k^{6 \alpha} \lambda^{4}-20 k^{\alpha} c^{\alpha}}}{30 k^{\alpha}}, \\
& a_{1}=-2 k^{2 \alpha} \lambda, \quad a_{2}=-2 k^{2 \alpha} .
\end{aligned}
$$

Substituting the result above into Eq. (31) and combining with (7), we can obtain corresponding exact solutions to Eq. (27).

When $\lambda^{2}-4 \mu>0$,

$$
\begin{aligned}
u_{1}(x, t)= & -\frac{40 k^{3 \alpha} \mu+5 k^{3 \alpha} \lambda^{2} \pm \sqrt{80 k^{6 \alpha} \mu^{2}-40 k^{6 \alpha} \mu \lambda^{2}+5 k^{6 \alpha} \lambda^{4}-20 k^{\alpha} c^{\alpha}}}{30 k^{\alpha}} \\
& -2 k^{2 \alpha} \lambda\left[-\frac{\lambda}{2}+\frac{\sqrt{\lambda^{2}-4 \mu}}{2}\left(\frac{C_{1} \sinh \frac{\sqrt{\lambda^{2}-4 \mu}}{2 \Gamma(1+\alpha)} \xi^{\alpha}+C_{2} \cosh \frac{\sqrt{\lambda^{2}-4 \mu}}{2 \Gamma(1+\alpha)} \xi^{\alpha}}{C_{1} \cosh \frac{\sqrt{\lambda^{2}-4 \mu}}{2 \Gamma(1+\alpha)} \xi^{\alpha}+C_{2} \sinh \frac{\sqrt{\lambda^{2}-4 \mu}}{2 \Gamma(1+\alpha)} \xi^{\alpha}}\right)\right] \\
& -2 k^{2 \alpha}\left[-\frac{\lambda}{2}+\frac{\sqrt{\lambda^{2}-4 \mu}}{2}\left(\frac{C_{1} \sinh \frac{\sqrt{\lambda^{2}-4 \mu}}{2 \Gamma(1+\alpha)} \xi^{\alpha}+C_{2} \cosh \frac{\sqrt{\lambda^{2}-4 \mu}}{2 \Gamma(1+\alpha)} \xi^{\alpha}}{C_{1} \cosh \frac{\sqrt{\lambda^{2}-4 \mu}}{2 \Gamma(1+\alpha)} \xi^{\alpha}+C_{2} \sinh \frac{\sqrt{\lambda^{2}-4 \mu}}{2 \Gamma(1+\alpha)} \xi^{\alpha}}\right)\right]^{2},
\end{aligned}
$$

where $\xi=k x+c t+\xi_{0}$

When $\lambda^{2}-4 \mu<0$,

$$
\begin{aligned}
u_{2}(x, t)= & -\frac{40 k^{3 \alpha} \mu+5 k^{3 \alpha} \lambda^{2} \pm \sqrt{80 k^{6 \alpha} \mu^{2}-40 k^{6 \alpha} \mu \lambda^{2}+5 k^{6 \alpha} \lambda^{4}-20 k^{\alpha} c^{\alpha}}}{30 k^{\alpha}} \\
& -2 k^{2 \alpha} \lambda\left[-\frac{\lambda}{2}+\frac{\sqrt{4 \mu-\lambda^{2}}}{2}\left(\frac{-C_{1} \sin \frac{\sqrt{4 \mu-\lambda^{2}}}{2 \Gamma(1+\alpha)} \xi^{\alpha}+C_{2} \cos \frac{\sqrt{4 \mu-\lambda^{2}}}{2 \Gamma(1+\alpha)} \xi^{\alpha}}{C_{1} \cos \frac{\sqrt{4 \mu-\lambda^{2}}}{2 \Gamma(1+\alpha)} \xi^{\alpha}+C_{2} \sin \frac{\sqrt{4 \mu-\lambda^{2}}}{2 \Gamma(1+\alpha)} \xi^{\alpha}}\right)\right] \\
& -2 k^{2 \alpha}\left[-\frac{\lambda}{2}+\frac{\sqrt{4 \mu-\lambda^{2}}}{2}\left(\frac{-C_{1} \sin \frac{\sqrt{4 \mu-\lambda^{2}}}{2 \Gamma(1+\alpha)} \xi^{\alpha}+C_{2} \cos \frac{\sqrt{4 \mu-\lambda^{2}}}{2 \Gamma(1+\alpha)} \xi^{\alpha}}{C_{1} \cos \frac{\sqrt{4 \mu-\lambda^{2}}}{2 \Gamma(1+\alpha)} \xi^{\alpha}+C_{2} \sin \frac{\sqrt{4 \mu-\lambda^{2}}}{2 \Gamma(1+\alpha)} \xi^{\alpha}}\right)\right]^{2},
\end{aligned}
$$

where $\xi=k x+c t+\xi_{0}$.

When $\lambda^{2}-4 \mu=0$,

$$
\begin{aligned}
u_{3}(x, t)= & -\frac{40 k^{3 \alpha} \mu+5 k^{3 \alpha} \lambda^{2} \pm \sqrt{80 k^{6 \alpha} \mu^{2}-40 k^{6 \alpha} \mu \lambda^{2}+5 k^{6 \alpha} \lambda^{4}-20 k^{\alpha} c^{\alpha}}}{30 k^{\alpha}} \\
& -2 k^{2 \alpha} \lambda\left[-\frac{\lambda}{2}+\frac{C_{2} \Gamma(1+\alpha)}{C_{1} \Gamma(1+\alpha)+C_{2} \xi^{\alpha}}\right] \\
& -2 k^{2 \alpha}\left[-\frac{\lambda}{2}+\frac{C_{2} \Gamma(1+\alpha)}{C_{1} \Gamma(1+\alpha)+C_{2} \xi^{\alpha}}\right]^{2}
\end{aligned}
$$

where $\xi=k x+c t+\xi_{0}$ 
Remark 6 The established solutions in Eqs. (32)-(34) are new exact solutions for the space-time fractional fifth-order Sawada-Kotera equation.

\section{Conclusions}

We have proposed a new fractional sub-equation method for solving FPDEs successfully, which is the fractional version of the known $\left(G^{\prime} / G\right)$ method. As one can see, the two nonlinear fractional complex transformations for $\xi$ and $\eta$ used here are very important. The first transformation ensures that a certain fractional partial differential equation can be turned into another fractional ordinary differential equation, the solutions of which can be expressed by a polynomial in $\left(\frac{D_{\xi}^{\alpha} G}{G}\right)$, where $G$ satisfies the fractional ODE $D_{\xi}^{2 \alpha} G(\xi)+\lambda D_{\xi}^{\alpha} G(\xi)+\mu G(\xi)=0$. The general expression for $\left(\frac{D_{\xi}^{\alpha} G}{G}\right)$ related to this fractional ODE can be obtained due to the second fractional complex transformations for $\eta$. Finally, we note that with this kind of nonlinear fractional complex transformations, it is worth to investigate the applications of other algebraic methods to fractional partial differential equations such as the Exp-function method, F-expansion method, Jacobi elliptic function method and so on.

\section{Competing interests}

The authors declare that they have no competing interests.

\section{Authors' contributions}

BZ carried out the main part of this article. All authors read and approved the final manuscript.

\section{Acknowledgements}

The authors would like to thank the reviewers very much for their valuable suggestions on the paper.

Received: 10 November 2012 Accepted: 21 June 2013 Published: 5 July 2013

\section{References}

1. Saadatmandi, A, Dehghan, AM: A new operational matrix for solving fractional-order differential equations. Comput. Math. Appl. 59, 1326-1336 (2010)

2. Zhou, Y, Jiao, F, Li, J: Existence and uniqueness for p-type fractional neutral differential equations. Nonlinear Anal. 71, 2724-2733 (2009)

3. Galeone, L, Garrappa, R: Explicit methods for fractional differential equations and their stability properties. J. Comput. Appl. Math. 228, 548-560 (2009)

4. Trigeassou, JC, Maamri, N, Sabatier, J, Oustaloup, A: A Lyapunov approach to the stability of fractional differential equations. Signal Process. 91, 437-445 (2011)

5. Deng, W: Smoothness and stability of the solutions for nonlinear fractional differential equations. Nonlinear Anal. 72, 1768-1777 (2010)

6. Ghoreishi, F, Yazdani, S: An extension of the spectral Tau method for numerical solution of multi-order fractional differential equations with convergence analysis. Comput. Math. Appl. 61, 30-43 (2011)

7. Edwards, JT, Ford, NJ, Simpson, AC: The numerical solution of linear multi-term fractional differential equations: systems of equations. J. Comput. Appl. Math. 148, 401-418 (2002)

8. Muslim, M: Existence and approximation of solutions to fractional differential equations. Math. Comput. Model. 49, 1164-1172 (2009)

9. Jafari, H, Tajadodi, H, Baleanu, D, Al-Zahrani, AA, Alhamed, YA, Zahid, AH: Fractional sub-equation method for the fractional generalized reaction Duffing model and nonlinear fractional Sharma-Tasso-Olver equation. Cent. Eur. J. Phys. (2013). doi:10.2478/s11534-013-0203-7

10. Baleanu, D, Rezapour, S, Mohammadi, H: Some existence results on nonlinear fractional differential equations. Philos. Trans. R. Soc. A, Math. Phys. Eng. Sci. 371(1990), 20120144 (2013)

11. Nyamoradi, N, Baleanu, D, Agarwal, RP: On a multipoint boundary value problem for a fractional order differential inclusion on an infinite interval. Adv. Math. Phys. 2013, Article ID 823961 (2013)

12. El-Sayed, AMA, Gaber, M: The Adomian decomposition method for solving partial differential equations of fractal order in finite domains. Phys. Lett. A 359, 175-182 (2006)

13. El-Sayed, AMA, Behiry, SH, Raslan, WE: Adomian's decomposition method for solving an intermediate fractional advection-dispersion equation. Comput. Math. Appl. 59, 1759-1765 (2010)

14. Daftardar-Gejji, V, Jafari, H: Adomian decomposition: a tool for solving a system of fractional differential equations. J. Math. Anal. Appl. 301(2), 508-518 (2005)

15. He, JH: A new approach to nonlinear partial differential equations. Commun. Nonlinear Sci. Numer. Simul. 2, 230-235 (1997)

16. Wu, G, Lee, EWM: Fractional variational iteration method and its application. Phys. Lett. A 374, 2506-2509 (2010) 
17. Guo, S, Mei, L: The fractional variational iteration method using He's polynomials. Phys. Lett. A 375, 309-313 (2011)

18. Wu, GC, Baleanu, D: Variational iteration method for the Burgers' flow with fractional derivatives - new Lagrange multipliers. Appl. Math. Model. 37, 6183-6190 (2013)

19. Jafari, $H$, Tajadodi, $H$, Baleanu, D: A modified variational iteration method for solving fractional Riccati differential equation by Adomian polynomials. Fract. Calc. Appl. Anal. 16(1), 109-122 (2013)

20. Jafari, H, Kadem, A, Baleanu, D, Yilmaz, T: Solutions of the fractional Davey-Stewartson equations with variational iteration method. Rom. Rep. Phys. 64(2), 337-346 (2012)

21. Jafari, $H$, Khalique, $C M$ : Analytical solutions of nonlinear fractional differential equations using variational iteration method. J. Nonlinear Syst. Appl. 2(3-4), 148-151 (2011)

22. Jafari, H, Tajadodi, H: He's variational iteration method for solving fractional Riccati differential equation. Int. J. Differ Equ. 2010, Article ID 764738 (2010)

23. He, JH: Homotopy perturbation technique. Comput. Methods Appl. Mech. Eng. 178, 257-262 (1999)

24. He, JH: A coupling method of homotopy technique and a perturbation technique for non-linear problems. Int. J. Non-Linear Mech. 35, 37-43 (2000)

25. Ganji, ZZ, Ganji, DD, Jafari, H, Rostamian, M: Application of the homotopy perturbation method for coupled system of partial differential equations with time fractional derivatives. Topol. Methods Nonlinear Anal. 31(2), $341-348$ (2008)

26. Jafari, H, Momani, Sh: Solving fractional diffusion and wave equations by modified homotopy perturbation method. Phys. Lett. A 370, 388-396 (2007)

27. Odibat, Z, Momani, S: Fractional Green function for linear time-fractional equations of fractional order. Appl. Math. Lett. 21, 194-199 (2008)

28. Cui, M: Compact finite difference method for the fractional diffusion equation. J. Comput. Phys. 228, 7792-7804 (2009)

29. Huang, Q, Huang, G, Zhan, H: A finite element solution for the fractional advection-dispersion equation. Adv. Water Resour. 31, 1578-1589 (2008)

30. Zhang, S, Zhang, HQ: Fractional sub-equation method and its applications to nonlinear fractional PDEs. Phys. Lett. A 375, 1069-1073 (2011)

31. Guo, SM, Mei, LQ, Li, Y, Sun, YF: The improved fractional sub-equation method and its applications to the space-time fractional differential equations in fluid mechanics. Phys. Lett. A 376(2012), 407-411 (2012)

32. Lu, B: Bäcklund transformation of fractional Riccati equation and its applications to nonlinear fractional partial differential equations. Phys. Lett. A 376, 2045-2048 (2012)

33. Lu, B: The first integral method for some time fractional differential equations. J. Math. Anal. Appl. 395, 684-693 (2012)

34. Zheng, $B$ : $\left(G^{\prime} / G\right)$-Expansion method for solving fractional partial differential equations in the theory of mathematical physics. Commun. Theor. Phys. 58, 623-630 (2012)

35. Jumarie, G: Modified Riemann-Liouville derivative and fractional Taylor series of nondifferentiable functions further results. Comput. Math. Appl. 51, 1367-1376 (2006)

36. Wang, ML, Li, XZ, Zhang, JL: The (G'/G)-expansion method and travelling wave solutions of nonlinear evolution equations in mathematical physics. Phys. Lett. A 372, 417-423 (2008)

37. Wang, ML, Zhang, JL, Li, XZ: Application of the ( $\left.\mathrm{G}^{\prime} / \mathrm{G}\right)$-expansion to travelling wave solutions of the Broer-Kaup and the approximate long water wave equations. Appl. Math. Comput. 206, 321-326 (2008)

38. Aslan, I: Discrete exact solutions to some nonlinear differential-difference equations via the ( $\left.\mathrm{G}^{\prime} / \mathrm{G}\right)$-expansion method. Appl. Math. Comput. 215, 3140-3147 (2009)

39. Ayhan, B, Bekir, A: The ( $\left.G^{\prime} / G\right)$-expansion method for the nonlinear lattice equations. Commun. Nonlinear Sci. Numer. Simul. 17, 3490-3498 (2012)

40. Kong, CC, Wang, D, Song, LN, Zhang, HQ: New exact solutions to MKDV-Burgers equation and ( $2+1)$-dimensional dispersive long wave equation via extended Riccati equation method. Chaos Solitons Fractals 39, 697-706 (2009)

41. Yan, ZY: Generalized transformations and abundant new families of exact solutions for $(2+1)$-dimensional dispersive long wave equations. Comput. Math. Appl. 46, 1363-1372 (2003)

42. Zhang, JF, Han, P: New multisoliton solutions of the $(2+1)$-dimensional dispersive long wave equations. Commun. Nonlinear Sci. Numer. Simul. 6, 178-182 (2001)

43. Eslami, M, Neyrame, A, Ebrahimi, M: Explicit solutions of nonlinear $(2+1)$-dimensional dispersive long wave equation. J. King Saud Univ, Comput. Inf. Sci. 24, 69-71 (2012)

44. Chen, Y, Wang, Q: A new general algebraic method with symbolic computation to construct new doubly-periodic solutions of the (2+1)-dimensional dispersive long wave equation. Appl. Math. Comput. 167, 919-929 (2005)

45. Wang, Q, Chen, Y, Zhang, HQ: An extended Jacobi elliptic function rational expansion method and its application to $(2+1)$-dimensional dispersive long wave equation. Phys. Lett. A 340, 411-426 (2005)

46. Chen, Y, Li, B: Symbolic computation and construction of soliton-like solutions to the $(2+1)$-dimensional dispersive long-wave equations. Int. J. Eng. Sci. 42, 715-724 (2004)

47. Liu, N, Liu, XQ, Lu, HL: New exact solutions and conservation laws of the $(2+1)$-dimensional dispersive long wave equations. Phys. Lett. A 373, 214-220 (2009)

48. Zhang, S, Tong, JL, Wang, W: Exp-function method for a nonlinear ordinary differential equation and new exact solutions of the dispersive long wave equations. Comput. Math. Appl. 58, 2294-2299 (2009)

49. Zhou, YQ, Liu, Q, Zhang, J, Zhang, WN: Exact solution for $(2+1)$-dimension nonlinear dispersive long wave equation. Appl. Math. Comput. 177, 495-499 (2006)

50. Yomba, E: The modified extended Fan's sub-equation method and its application to $(2+1)$-dimensional dispersive long wave equation. Chaos Solitons Fractals 26, 785-794 (2005)

51. Chen, Y, Wang, Q: A series of new soliton-like solutions and double-like periodic solutions of a $(2+1)$-dimensional dispersive long wave equation. Chaos Solitons Fractals 23, 801-807 (2005)

52. Zeng, $X$, Zeng, J: Symbolic computation and new families of exact solutions to the $(2+1)$-dimensional dispersive long-wave equations. Chaos Solitons Fractals 29, 1115-1120 (2006)

53. Zhang, S: The periodic wave solutions for the $(2+1)$-dimensional dispersive long water equations. Chaos Solitons Fractals 32, 847-854 (2007)

54. Liu, CF, Dai, ZD: Exact soliton solutions for the fifth-order Sawada-Kotera equation. Appl. Math. Comput. 206, 272-275 (2008) 
doi:10.1186/1687-1847-2013-199

Cite this article as: Zheng and Wen: Exact solutions for fractional partial differential equations by a new fractional sub-equation method. Advances in Difference Equations 2013 2013:199.

Submit your manuscript to a SpringerOpen ${ }^{\circ}$ journal and benefit from:

- Convenient online submission

- Rigorous peer review

- Immediate publication on acceptance

- Open access: articles freely available online

- High visibility within the field

- Retaining the copyright to your article

Submit your next manuscript at $\gg$ springeropen.com 